\title{
A METHOD OF FORECASTING EARTHQUAKE BY OPTICAL ASTRONOMIC TIME- LATITUDE RESIDUALS
}

\author{
Youjin SU ${ }^{1)}$, Yuping GAO ${ }^{2)}$, Chunxiao $\mathrm{LI}^{3)}$ and Hui $\mathrm{HU}^{3)}$ \\ ${ }^{1)}$ Seismological Bureau of Yunnan Province, Kunming 650224, P.R.China; e-mail: suyoujin0818@sina.com \\ 2) National Time Service Center, Chinese Academy of Sciences, Xi'an, Shaanxi 710600, P.R.China; e-mail: gaoyp@ntsc.ac.cn \\ 3) Yunnan Observatories, Chinese Academy of Sciences, Kunming 650216,P.R.China; e-mail:lcx@ynao.ac.cn; huhui@mail.ynao.ac.cn
}

\section{ARTICLE INFO}

Article history

Received 4 August 2017

Accepted 29 November 2017

Available online 21 December 2017

Keywords:

Optical astronomic time-latitude residuals Change of the plumb line

Motion of the groundmass

Earthquake

\begin{abstract}
This article reports a method for forecasting an earthquake by synchronous anomalies of optical astronomic time-latitude residuals. The so-called optical astronomic time-latitude residuals for a certain astrometric instrument are the rest after deducting the effects of Earth whole motion from the astronomical time and latitude observations determined by the instrument. Forecasting practice for four earthquakes around the Yunnan Observatory occurring after 2010 shows that it does not generate false forecasts, and also does not miss forecasts of major earthquakes. This forecasting practice proves that the synchronous anomalies of astronomical timelatitude residuals can provide effective warning sign for earthquake occurrence around observatory station, thus deserves attention and further study.
\end{abstract}

\section{LNTRODUCTION}

Earthquake prediction is a worldwide scientific difficult problem (Geller et al., 1997; Knopoff, 1992). Accurate optical astrometric observations not only may be used for the astronomical researches ( $\mathrm{Hu}$ et al., 1999; Hu and Wang, 2002), but also can provide important information for the earthquake forewarning. After occurrence of a great earthquake 7.8 in Tangshan region of China in 1976, the researchers of Beijing Astronomical Observatory of Chinese Academy of Sciences found a kind of short-term anomalous variation appeared in the astronomic timelatitude residuals of observations obtained by an astrolabe in the Shahe observational station just before the earthquake. In order to further confirm the objectivity, authenticity and universality of this phenomenon, they collected observation data of 11 observing stable and well-qualified instruments from 10 observatories in the world since 1966 and systematically analyzed them. It was found that there were no such synchronous anomalies in the observation results of years without earthquakes or the stations without earthquake, this kind of synchronous anomalies only appeared in years with earthquakes around the observation station. This affirms that this synchronous anomaly is associated with the earthquakes, and named astronomical time-latitude residuals short-term synchronous anomalies before strong earthquakes (Li et al., 1978). Through more than 40 years study by researchers from the Beijing Astronomical Observatory, the Shanghai Observatory and the Yunnan Observatory, significant synchronous anomalies of optical astronomical time-latitude residuals were found and observed within 2-3 months before occurrence of earthquakes $M \geq 6.0$ occurring within $700 \mathrm{~km}$ around the observatories, and vice versa no such synchronous anomalies were observed (Han et al., 2003; Han., 1993; Han and Tian, 1993; Han et. al., 2012; Hu et al., 1994; $\mathrm{Hu}$ et al., 1996; $\mathrm{Hu}$ and Han, 1997; Hu et al., 2007; Hu et al., 2003; Kan and $\mathrm{Hu}, 1995$; Liao et al., 1994; Liao and Zheng, 1990). The result has been submitted to the 19th Generally Assembly of the International Astronomical Union (IAU) in 1985. The 19th Committee (Earth Rotation Committee) pointed out that optical astrometric observations of latitude and universal time might be valuable for studying geophysical phenomena and the variation of the plumb line (Swing, 1985; Kotaczel, 1988; Bergeron, 1991). The optical astronomical time-latitude residuals for a certain astrometric instrument are rest after deducting the effects of Earth rotation (rotational rate and polar motion) from the astronomical time and latitude observations determined by the optical astrometric instrument. The optical astrometric instruments take the local plumb line as the observation reference. Its reason of anomaly is possibly from change of the plumb line due to the motion of the groundmass before 
earthquake (Zhang, 1981). The so-called anomaly is change of the residuals beyond the normal range of error, we only take errors greater than twice the standard deviation as an anomaly. Although this phenomenon, i.e. optical astronomy time-latitude residuals short-term synchronous anomalies appearing before strong earthquakes, was discovered in the last century ( $\mathrm{Li}$ et al., 1978), but subsequent studies basically still remained as analysis after the occurrence of earthquakes, only from 2010 we formally systematically include it in the work of preearthquake predictions. Since 2010 practice of prediction shows that this method is reliable, worthy of attention and promotion.

\section{FORECASTING METHOD} praktice

The Forecasting method is data processing in 1. According to definition of the optical astronomical time-latitude residuals calculate initial values of the time residual $R T_{j}$ and latitude residual $R L_{j}$ determined by an instrument $j$. It may be respectively express as:

$\mathrm{RT}^{\prime}{ }_{\mathrm{i}}=(\mathrm{UT} 0-\mathrm{UTC})_{\mathrm{i}}+\Delta \lambda-(\mathrm{UT} 1-\mathrm{UTC})_{\mathrm{i}}$

$\mathrm{RL}^{\prime}{ }_{\mathrm{i}}=\mathrm{d} \Phi_{\mathrm{i}}-\mathrm{d} \Phi_{\mathrm{c}}$

Here (UT0-UTC) is the original value of universal time obtained by the instrument; $\Delta \lambda$ is the correctional value, which is related to the polar motion, from (UT0-UTC) system to (UT1-UTC) system. (UT1-UTC) is value of universal time of the date. $d \Phi_{j}$ and $d \Phi_{c}$ are respectively the variational value of the latitude determined by the instrument and the polar term of latitude variation of homologous time. $\Delta \lambda_{j}$ and $d \Phi_{c}$ may be respectively expressed as the following formulae:

$$
\begin{aligned}
& \Delta \lambda=\frac{1}{15}\left(X \sin \lambda_{j}-Y \cos \lambda_{j}\right) \operatorname{tg} \Phi_{j} \\
& d \Phi_{c}=X \cos \lambda_{j}+Y \sin \lambda_{j}
\end{aligned}
$$

Here $X, Y$ is the coordinate of the Earth's pole of homologous time. $\lambda_{j}$ and $\Phi_{j}$ are respectively the longitude and latitude of the instrument. (UT1-UTC), $X$ and $Y$ are provided by the International Earth Rotation Service (IERS). The time residual $R T_{j}$ and latitude residual $R L_{j}$ of a certain instrument will be obtained through its observations and the formulae.

2. Reduce to zero the mean values of the two sequences $R T$ and $R F$ respectively.

$$
\begin{array}{ll}
T_{0}=\frac{1}{N} \sum_{i=1}^{N} R T_{i}^{\prime} & R T^{\prime \prime}{ }_{i}=R T^{\prime}{ }_{i}-T_{0} \\
L_{0}=\frac{1}{N} \sum_{i=1}^{N} R L_{i}^{\prime} & R L^{\prime \prime}{ }_{i}=R L^{\prime}{ }_{i}-L_{0}
\end{array}
$$

3. According to the analyses made by astronomers for many years, there generally exist local terms with annual and semi-annual harmonic variations in the observations of time and latitudes obtained at all the stations over the world (Kimura, 1902; BIH, 1984). So after making harmonic analyses of $R T$ and $R F$, the expressions of their local terms may be found as follows:

(a) The sinusoidal curve mirroring mean variation of $R T^{\prime \prime}$ and $R L^{\prime \prime}$ is respectively fitted according to the following formula,

$A \sin (2 \pi t+\alpha)+B \sin (4 \pi t+\beta)$,

Here $A$ and $B$ are the coefficients of annual and semiannual terms respectively, $\alpha$ and $\beta$ are the phases of the two terms respectively, $t$ is time calculated from the initial date of the calendar year.

(b) The local terms are deducted respectively from the sequences of $R T^{\prime \prime}$ and $R L^{\prime \prime}$, thereby obtaining the purified astronomical time and latitude residual sequences $R T$ and $R L$.

4. Calculate the weighted mean values for intervals of five days for $R T$ and $R F$, respectively.

5. In order to eliminate short term accidental disturbance, take the five-point weighted running mean for each sequence.

$\mathrm{S}_{\mathrm{i}}=\frac{1}{9}\left(\mathrm{~F}_{\mathrm{i}-2}+2 \mathrm{~F}_{\mathrm{i}-1}+3 \mathrm{~F}_{\mathrm{i}}+2 \mathrm{~F}_{\mathrm{i}+1}+\mathrm{F}_{\mathrm{i}+2}\right)$

Here F represents RT or RL.

6. Calculate the standard deviation $\sigma$ for each sequence and then draw the diagram: $\pm|2 \sigma|$ are taken as the limits of the anomaly.

$\sigma=\sqrt{\frac{\sum S^{2}-\frac{\left(\sum S\right)^{2}}{n}}{n-1}}$

\section{FORECASTING PRACTICE SINCE 2010}

Since February of 2010, at the beginning of each month we have provided a report to the Earthquake Prediction Research Center of Yunnan Province listing the dates of synchronous anomalies point of a value of each 5 days of astronomical time-latitude residuals until end of proceeding, for forecasting research purposes. From the monthly reported data in 2010-2015 the observational situation of photoelectric astrolabe of the Yunnan Observatory was very good. Besides synchronous anomalies in March of 2013 and March, May and September of 2014 before earthquakes, there were no other synchronous anomalies (see Fig. 1). Therefore from the situation in 2010-2015 there was neither false nor fail report.

Two synchronous anomalies among the four observed synchronous anomalies appeared before the Yingjiang earthquake 6.1 on 2014-05-30. The second 

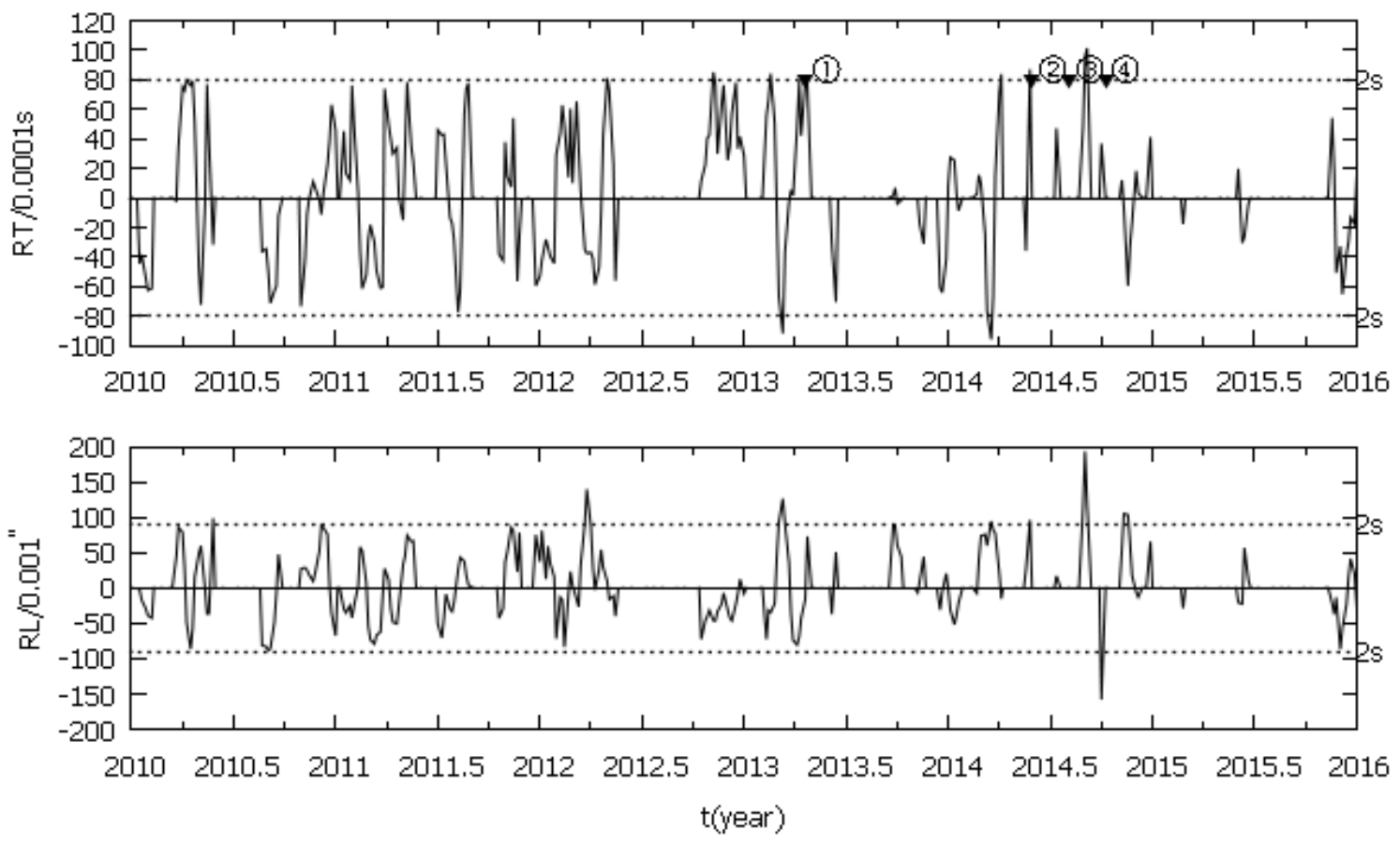

Fig. 1 Residuals curves of astronomical time (a) and latitude (b) of photoelectric astrolabe of Yunnan Observatory from 2010.0 to 2016.05

(1)2013-04-20, Lusha 7.0 ; (2)2014-05-30, Yingjiang 6.1 ;

(3)2014-08-03, Ludian 6.5 ; (4)2014-10-07, Jinggu 6.6。

anomaly occurred just a few days before the Yingjiang earthquake on 2014-05-30. Why we believe that the anomaly in March of 2014 corresponds to the Yingjiang earthquake on 2014-05-30, and the other anomaly in May corresponds to the Ludian earthquake 6.5 on 2014-08-03? This is based on our previous research for many astrometric observations over the world. We found that the synchronous anomalies of astronomical time-latitude residuals generally appear weeks to several months before the earthquake. For the synchronous anomalies of astronomic timelatitude residuals of the photoelectric astrolabe of the Yunnan Observatory and the strong earthquakes around it, the time lag generally is 2-3 months. Therefore based on above-mentioned analyses we think that the synchronous anomalies on March and May of 2014 most likely respectively correspond to the Yingjiang earthquake 6.1 on 2014-05-30 and the Ludian earthquake 6.5 on 2014-08-03. In Figure 1, in addition to the four synchronous anormalies abovementioned, there are sometimes a few exceptions, non-synchronous anormaly, i.e. they are only a single time residual anormaly, or a single latitude residual anormaly, according to the current study, such type of single anormaly is unrelated to earthquakes.

The distances from the epicenters of the Lushan, Yingjiang, Ludian and Jinggu earthquakes to the Yunnan Observatory are respectively $580 \mathrm{~km}$, $480 \mathrm{~km}, 240 \mathrm{~km}$ and $280 \mathrm{~km}$, their orientations are respectively 12 degrees north east, 10 degrees north west, north east of 20 degrees and 48 degrees south west (Fig. 2). Upon appearing of the four synchronous anomalies before the four time earthquakes, we provided reports on the synchronous anomalies of astronomical time-latitude residuals to the Earthquake Prediction Research Center of the Yunnan Province before these four earthquakes. This proves that the synchronous anomalies of astronomical time-latitude residuals can provide effective warning sign for earthquake occurrence around observatory station, thus deserves attention and further study.

\section{DISCUSSION}

Because our photoelectric astrolabe uses a vacuum tube, the star light enters the vacuum tube after refraction passing through vacuum space and variety refraction passing through the Earth's atmosphere, because the two refraction reflex, i.e. passing through vacuum space and passing through atmosphere are opposite, the refraction in the entire path of the star light from vacuum space to the atmosphere and from atmosphere to the vacuum tube is basically eliminated.

From the data in Figure 1, the continuity of curve of astronomical time-latitude residual is not good. This is because the observation of the optical astrometric instruments is carried out only in the night of sunny day with few clouds. In Kunming, however, every year from May to October is the rainy season, which seriously affects the continuity of optical astronomical time-latitude residuals data. 


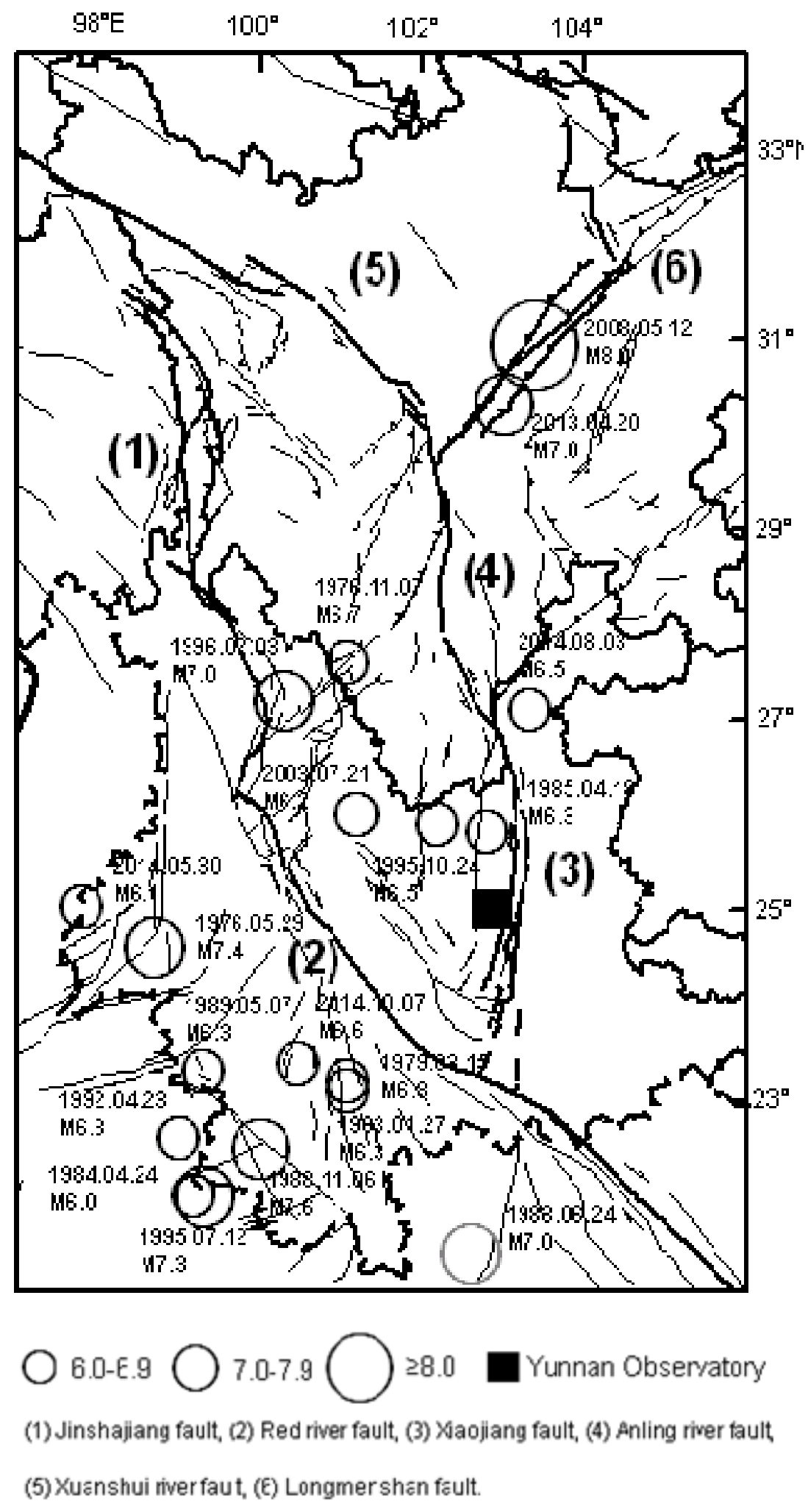

Fig. 2 Geological structure of the Xikang-Yunnan fault block.

In Figure 2, we mark the date and magnitude of the earthquakes but not the locality because many earthquakes have occurred in the same location. 
Forecasting earthquake by the synchronous anormalies of optical astronomical time-latitude residuals at present can still only give accurate early warning information. There will be forecast a strong earthquake of $M \geq 6.0$ occurred within $700 \mathrm{~km}$ around Observatory in the next 2-3 months. Since development and occurrence of earthquakes are extremely complex geophysical processes, accurate forecasts cannot be obtained by any single means. In order to accurately forecast three earthquake elements (time, location and magnitude), the comprehensive analysis by multiple methods and observations has to be taken.

\section{ACKNOWLEDGEMENTS}

We extend heartfelt thanks to the referees for their valuable comments. IERS (the International Earth Rotation and Reference Systems Service) provide high precision Earth Orientation Parameters for a long time. Dr. Chen I-wan, former advisor of the Committee of Natural Hazard Prediction of China Geophysics Society provided us valuable discussion and help. We extend our heartfelt thanks to them.

The photoelectric astrolabe is jointly operated and administrated by the Yunnan Observatory and the Center for Astronomical Mega-Science, Chinese Academy of Sciences. This work is supported by the tackle key project of the Ministry of Science and Technology of P. R. China (2012BAK19B01-07), and National Time Service Center and Center for Astronomical Mega-Science, Chinese Academy of Sciences.

\section{REFERENCES}

Bergeron, J. (Ed.): 1991, Transactions of the International Astronomical Union, XXIB. Proc. 21st General Assembly, Buenos Aires, Kluwer Academic Publishers, 209 pp.

BIH (Bureau International de l'Heure): 1984, Annual Report for 1983, Paris.

Geller, R.J., Jackson, D.D., Kagan, Y.Y. and Mulargia, F.: 1997, Earthquakes cannot be predicted. Science, 275, 1616-1617.

Han, Y.B.: 1993, Relationship between residual anomalies of time-latitude and earthquakes. Chin. Sci. Bull., 38, 4, 303-307.

Han, Y.B, Guo, Z.J. and Wu, J.B: 2003, Possible triggering of solar activity to big earthquake $(\mathrm{M} \geq 8.0)$ in faults with near west-east strike in China. Science in China, Series G-Physics, Mechanics \&Astronomy, 33, 6, 567-573.

Han, Y.B., Ma, L. and Hu H. et al.: 2007, Application of astronomic time-latitude residuals in earthquake prediction. Earth, Moon and Planets, 100, 1, 125-135. DOI: $10.1007 / \mathrm{s} 11038-006-9125-3$

Han, Y.B. and Tian, J.: 1993, Method and application software providing information on earthquake prediction with the classical astrometric observations. Publ. Beijing Astron. Obs., 22, 41-44.

Han,Y.B., Wang, H.Q, Yin, Z.Q. et al.: 2012, Significance and feasibility to set up test measurement networks in Huabei and Xibei regions for variation of the vertical. Prog. Geophys., 27, 4, 1287-1293, (in Chinese).
Hu, H. and Han, Y.B.: 1997, Application of the anomaly of astrometric observations to earthquake prediction study. In: Forsberg, R., Feissel, $\mathrm{M}$ and Dietrich. R. (Eds.), International Association of Geodesy Symposia, IAG Scientific Assembly, Rio de Janeiro, 119, Springer Press, 460-464.

Hu, H., Kan, R.J. and Li, X.M.: 1996, Application of the residuals of astronomic time and latitude in the earthquake prediction. Chin. Sci. Bull., 41, 13, 1104 1106.

Hu, H., Li, Z.X., Li, H. and Fu, G.Y: 2003, Internnual variations of the vertical at Yunna by astrometry and gravimetry techniques. J. Natur. Disaster, 12, 2, 2527, (in Chinese).

Hu, H., Su, Y.J., Fu, H., Han, Y.B., Wang, R. and Ma, L.H.: 2007, Anomalies in time-latitude residuals at Yunnan Observatory before Dayao and Puer earthquakes. J. Natur. Disaster., 16, 5, 106-110, (in Chinese).

$\mathrm{Hu}, \mathrm{H}$. and Wang, R.: 2002, Optical positions of 55 radio stars from astrolabe observations from the Yunnan Observatory. A\&A, 383, 3, 1062-1066. DOI: 10.1051/0004-6361:20011831

Hu, H., Wang, R., Kan, R.J. and Zhao, H.S.: 1994, Occurrence of the short-term anomaly of residuals of astronomic time-latitude at Yunnan Observatory before the Puer earthquake. Proc. International Workshop on Seismotectonics and Seismic Hazard in South East Asia, Hanoi-Vietnam, 52-56.

$\mathrm{Hu}, \mathrm{H}$. , Wang, R. and Li, X.M.: 1999, Optical positions of 44 radio stars from astrolabe observations. Astron. J., 117, 6, 3066-3069. DOI: 10.1086/300894

Kan, R.J. and Hu, H.: 1995, The major earthquakes in and around Yunnan and variation of astronomical timelatitude residuals determined in Yunnan Observatory. Earthq. Res. China, 11, 4, 299-309, (in Chinese).

Kimura, H.: 1902, A new annual term in the variation of latitude, independent of the components of the Pole's motion. Astron. J., 22, 107-108.

Knopoff, L.: 1992, Earthquake prediction is difficult but not impossible. Nature Debate Sci., 39, 1633-1658.

Kotaczel, B. (Ed.): 1988, Transactions of the International Astronomical Union, XXB. Proc. 19th General Assembly, Baltimore, Kluwer Academic Publishers, 197 pp.

Li, Z.S., Zhang, G.D., Zhang, H.Z. and Liu, X.J.: 1978, Correlation between the short-term anomalies of residuals of astronomical time and latitude and major earthquakes around the observatories. Acta Geophys. Sin., 21, 4, 278-291, (in Chinese).

Liao, D.C., Jin, W.J., Zheng, D.W. and Wang, S.H.: 1994, An analysis of optical observations in China during 1986 to 1991. Ann. Shanghai Observatory Acad. Sin., 15, 28-33, (in Chinese).

Liao, D.C. and Zheng, D.W.: 1990, Earthquake $\left(\mathrm{M}_{\mathrm{S}}=5\right)$ in Taicang and anomalous residuals of time determination at Shanghai Observatory. Chin. Sci. Bull., 35, 23, 1838-1839.

Swing, J.P. (Ed.): 1985, Transactions of the International Astronomical Union, Vol. XIXB. Proc. 19th General Assembly, Delhi, Kluwer Academic Publishers, 175 pp.

Zhang, G.D.: 1981, The variation in the vertical line caused by the activity of the underground water before a strong earthquake. Acta Seismol. Sinica , 3, 2, 152158, (in Chinese). 